Pacific Journal of Mathematics

POWER-CANCELLATION OF GROUPS AND MODULES 


\title{
POWER-CANCELLATION OF GROUPS AND MODULES
}

\author{
K. R. GoodearL
}

This paper is concerned with deriving conditions which ensure that even though a module $A$ may not necessarily cancel from a direct sum $A \oplus B \cong A \oplus C$, it can at least be concluded that $B^{n} \cong C^{n}$ for some positive integer $n$. This conclusion is obtained from a type of stable range condition on the endomorphism ring of $A$, which holds, for example, when $A$ is a finitely generated module over any subring of a finite-dimensional Q-algebra. As an application of these methods to groups, it is shown that if $A$ is a torsion-free abelian group of finite rank, and $B, C$ are arbitrary groups (not necessarily abelian) such that $A \times B \cong A \times C$, then there exists a positive integer $n$ such that the direct product of $n$ copies of $B$ is isomorphic to the direct product of $n$ copies of $C$.

This research was partially supported by a National Science Foundation grant. The author would like to thank R. B. Warfield, Jr. for a number of very helpful conversations related to this material.

All rings in this paper are associative with unit, and all modules are unital. Most modules are right modules, so that endomorphism rings act on the left. The letter $R$ always denotes a ring.

1. Introduction. Let us say that a module $A$ satisfies the power-cancellation property if for all modules $B$ and $C, A \oplus B \cong A \oplus C$ implies that $B^{n} \cong C^{n}$ for some positive integer $n$. (Here $B^{n}$ denotes the direct sum of $n$ copies of $B$.) The objective of this paper is to derive sufficient conditions for power-cancellation. We obtain powercancellation from a stronger property called power-substitution: Given any decomposition $M=A_{1} \oplus B_{1}=A_{2} \oplus B_{2}$ with each $A_{i} \cong A$, there must exist a positive integer $n$ such that $B_{1}^{n}$ and $B_{2}^{n}$ have a common complement in $M^{n}$. Power-substitution depends only on the endomorphism ring of $A$, and is equivalent to a condition resembling the stable range conditions of algebraic $K$-theory. Because the derivation of power-cancellation from power-substitution is directly analogous to the derivation of cancellation theorems from the stable range conditions, we begin by recalling the appropriate stable range results.

Definition. A ring $R$ is said to have 1 in the stable range provided that whenever $a x+b=1$ in $R$, there exists $y \in R$ such that $a+b y$ is a unit in $R$. (It can be shown that this definition is left-right symmetric.) 
For example, every artinian ring has 1 in the stable range $[\mathbf{1 2}$, Lemma 11.8].

The following theorem shows that 1 in the stable range is equivalent to a substitution property. It was first proved by Fuchs for quasiprojective modules with projective covers [2, Theorem 3], then for arbitrary modules by Warfield [14].

Theorem 1.1. [14, Theorem 1] Let $A$ be a right $R$-module, and set $E=\operatorname{End}_{R}(A)$. Then $E$ has 1 in the stable range if and only if for any right $R$-module decomposition $M=A_{1} \oplus B_{1}=A_{2} \oplus B_{2}$ with each $A_{i} \cong A$, there exists a submodule $C \leqq M$ such that $M=C \oplus B_{1}=C \oplus B_{2}$.

The substitution property expressed in Theorem 1.1 clearly implies that $B_{1} \cong B_{2}$, which yields a proof of the following theorem.

THEOREM 1.2. [1, Theorem 2] Let $A$ be a right $R$-module such that $\operatorname{End}_{R}(A)$ has 1 in the stable range. If $B$ and $C$ are any right $R$-modules such that $A \oplus B \cong A \oplus C$, then $B \cong C$.

There are analogous substitution and cancellation results connected with the higher stable range conditions. Since some of these results will be needed later in the paper, we recall them here.

Definition. A row $\left(a_{1}, \cdots, a_{r}\right)$ of elements from a ring $R$ is said to be a right unimodular row if $\Sigma a_{i} R=R$. Given a positive integer $n$, a ring $R$ is said to have $n$ in the stable range provided that for any right unimodular row $\left(a_{1}, \cdots, a_{r}\right)$ of $r \geqq n+1$ elements of $R$, there exist elements $b_{1}, \cdots, b_{r-1} \in R$ such that the row $\left(a_{1}+a_{r} b_{1}, \cdots, a_{r-1}+a_{r} b_{r-1}\right)$ is right unimodular. (As above, this property can be shown to be left-right symmetric. Also, the case $n=1$ of this definition is easily seen to be equivalent to the previous definition of 1 in the stable range.)

Theorem 1.3. [14, Theorem 6] Let $A$ be a right $R$-module, set $E=\operatorname{End}_{R}(A)$, and let $n$ be a positive integer. Then $E$ has $n$ in the stable range if and only if for any right $R$-module decomposition

$$
M=A_{1} \oplus \cdots \oplus A_{n} \oplus B_{1}=A_{n+1} \oplus B_{2}
$$

with all $A_{i} \cong A$, there exist submodules $C \leqq M$ and $L \leqq A_{1} \oplus \cdots \oplus A_{n}$ such that $M=C \oplus L \oplus B_{1}=C \oplus B_{2}$.

THEOREM 1.4. [14, Theorem 7] Let $A$ be a right $R$-module such that $\operatorname{End}_{R}(A)$ has $n$ in the stable range, for some positive integer $n$. If $B$ and $C$ are any right $R$-modules such that $A \oplus B \cong A \oplus C$ and $B$ has a direct summand isomorphic to $A^{n}$, then $B \cong C$. 
2. Power-substitution. We use $M_{n}(R)$ to denote the ring of all $n \times n$ matrices over a ring $R$, and we use $I$ to denote the identity matrix in any $M_{n}(R)$. Given $r \in R$ and $P \in M_{n}(R)$, we write $r P$ and $P r$ for the matrices obtained from $P$ by multiplying each entry on the left (right) by $r$. In particular, $r I=I r$ is the diagonal matrix with all diagonal entries equal to $r$.

THEOREM 2.1. Let $A$ be a right $R$-module, and set $E=\operatorname{End}_{R}(A)$. Then the following conditions are equivalent:

(a) Given any right $R$-module decomposition $M=A_{1} \oplus B_{1}=$ $A_{2} \oplus B_{2}$ with each $A_{i} \cong A$, there exist a positive integer $n$ and a submodule $C \leqq M^{n}$ such that $M^{n}=C \oplus B_{1}^{n}=C \oplus B_{2}^{n}$.

(b) If $a x+b=1$ in $E$, then there exist a positive integer $n$ and $a$ matrix $Q \in M_{n}(E)$ such that $a I+b Q$ is a unit in $M_{n}(E)$.

Proof. Given any positive integer $n$, there is an additive functor from Mod- $R \rightarrow \operatorname{Mod}-R$ which carries any module $D$ to $D^{n}$. For any map $f: D \rightarrow E$, we use $f^{*}$ to denote the image of $f$ under this functor. Thinking of $f^{*}$ as an $n \times n$ matrix with entries from $\operatorname{Hom}_{R}(D, E), f^{*}$ is a diagonal matrix with all diagonal entries equal to $f$.

(a) $\Rightarrow$ (b): Set $M=A^{2}$, and let $p_{i}: M \rightarrow A, q_{i}: A \rightarrow M$ (for $i=1,2$ ) denote the projections and injections of this direct sum. Set $A_{1}=q_{1}(A)$ and $B_{1}=q_{2}(A)$, so that $M=A_{1} \oplus B_{1}$ with $A_{1} \cong A$. Define maps $f=$ $a p_{1}+b p_{2}$ from $M \rightarrow A$ and $g=q_{1} x+q_{2}$ from $A \rightarrow M$. Observing that $f g=a x+b=1_{A}$, we see that $M=g(A) \oplus(\operatorname{ker} f)$. Set $A_{2}=g(A)$ and $B_{2}=\operatorname{ker} f$, so that $M=A_{2} \oplus B_{2}$ with $A_{2} \cong A$.

According to (a), there exist $n>0$ and $C \leqq M^{n}$ such that $M^{n}=$ $C \oplus B_{1}^{n}=C \oplus B_{2}^{n}$. Since $C$ and $A_{1}^{n}$ are both complements for $B_{1}^{n}$ in $M^{n}$, we see that $C \cong A_{1}^{n} \cong A^{n}$. As a result, there exists a monomorphism $h: A^{n} \rightarrow M^{n}$ such that $h\left(A^{n}\right)=C$. Inasmuch as $p_{1}^{*}: M^{n} \rightarrow A^{n}$ is an epimorphism and

$$
M^{n}=C \oplus B_{1}^{n}=h\left(A^{n}\right) \oplus\left(\operatorname{ker} p_{1}^{*}\right),
$$

we infer that $p_{1}^{*} h: A^{n} \rightarrow A^{n}$ is an isomorphism. Similarly, $f^{*}$ is an epimorphism and

$$
M^{n}=C \oplus B_{2}^{n}=h\left(A^{n}\right) \oplus\left(\operatorname{ker} f^{*}\right),
$$

whence $f^{*} h$ is an isomorphism. Observing that $f^{*} h=a^{*} p_{1}^{*} h+b^{*} p_{2}^{*} h$, we conclude that $a^{*}+b^{*} p_{2}^{*} h\left(p_{1}^{*} h\right)^{-1}$ is an automorphism of $A^{n}$.

Identifying $\operatorname{End}_{R}\left(A^{n}\right)$ with $M_{n}(E)$ in the obvious manner, we thus have $Q=p_{2}^{*} h\left(p_{1}^{*} h\right)^{-1}$ in $M_{n}(E)$ such that $a I+b Q=a^{*}+b^{*} Q$ is a unit in $M_{n}(E)$. 
(b) $\Rightarrow$ (a): Using the decomposition $M=A_{1} \oplus B_{1} \cong A \oplus B_{1}$, we obtain projections $p_{1}: M \rightarrow A, p_{2}: M \rightarrow B_{1}$ and injections $q_{1}: A \rightarrow M$, $q_{2}: B_{1} \rightarrow M$ such that $p_{1} q_{1}=1_{A}, q_{1} p_{1}+q_{2} p_{2}=1_{M}$, and ker $p_{1}=B_{1}$. Using the decomposition $M=A_{2} \oplus B_{2} \cong A \oplus B_{2}$, we obtain a projection $f: M \rightarrow A$ and an injection $g: A \rightarrow M$ such that $f g=1_{A}$ and $\operatorname{ker} f=B_{2}$.

Now $1_{A}=f\left(q_{1} p_{1}+q_{2} p_{2}\right) g=\left(f q_{1}\right)\left(p_{1} g\right)+\left(f q_{2} p_{2} g\right)$. Setting $a=f q_{1}, x=$ $p_{1} g$, and $b=f q_{2} p_{2} g$, we thus have $a, x, b \in E$ such that $a x+b=1$. According to (b), there exist $n>0$ and $Q \in M_{n}(E)$ such that $a I+b Q$ is a unit in $M_{n}(E)$. Using the identification of $M_{n}(E)$ with $\operatorname{End}_{R}\left(A^{n}\right)$, we thus obtain a map $h: A^{n} \rightarrow A^{n}$ such that $\left(f^{*} q_{1}^{*}\right)+\left(f^{*} q_{2}^{*} p_{2}^{*} g^{*}\right) h=a^{*}+b^{*} h$ is an automorphism of $A^{n}$.

Set $k=q_{1}^{*}+q_{2}^{*} p_{2}^{*} g^{*} h: A^{n} \rightarrow M^{n}$ and $C=k\left(A^{n}\right)$. Since $f^{*} k$ is an isomorphism, we infer that $M^{n}=k\left(A^{n}\right) \oplus\left(\operatorname{ker} f^{*}\right)=C \oplus B_{2}^{n}$. Similarly, $p_{1}^{*} k=p_{1}^{*} q_{1}^{*}$ is the identity map on $A^{n}$, whence $M^{n}=$ $k\left(A^{n}\right) \oplus\left(\operatorname{ker} p_{1}^{*}\right)=C \oplus B_{1}^{n}$.

Definition. We say that a right $R$-module $A$ has the powersubstitution property if $A$ satisfies condition (a) of Theorem 2.1. We say that a ring $E$ has the right power-substitution property if the right module $E_{E}$ has the power-substitution property, or, equivalently, if $E$ satisfies condition (b) of Theorem 2.1. Obviously there is a left power-substitution property as well, but we do not know whether it is equivalent to right power-substitution.

Corollary 2.2. Let $A$ be a right $R$-module such that $\operatorname{End}_{R}(A)$ has the right power-substitution property. If $B$ and $C$ are any right $R$-modules such that $A \oplus B \cong A \oplus C$, then $B^{n} \cong C^{n}$ for some positive integer $n$.

Obviously any ring which has 1 in the stable range also satisfies right power-substitution, and there is a sense in which power-substitution and stable range 1 are nearly equivalent. Given a ring $R$ and positive integers $k, n$ such that $k \mid n$, there is a natural ring map $M_{k}(R) \rightarrow M_{n}(R)$. Considering the positive integers as a directed set ordered by divisibility, we thus obtain a directed system of matrix rings over $R$, and we can form the direct limit $S=\lim M_{n}(R)$. It is clear from the definitions that $S$ has 1 in the stable range if and only if every $M_{n}(R)$ satisfies right powersubstitution. It it were proved that right power-substitution is preserved in matrix rings, this would show that $R$ satisfies right power-substitution if and only if $S$ has stable range 1. (In addition, because of the left-right symmetry of stable range 1 , it would follow that power-substitution is left-right symmetric.)

In general, power-substitution is weaker than stable range 1. For example, $\mathbf{Z}$ has power-substitution (Corollary 3.4 ), but it is easily checked 
that $\mathbf{Z}$ does not have 1 in the stable range. More generally, if $F$ is any algebraic field extension of $\mathbf{Q}$, then every subring of $F$ satisfies powersubstitution (Corollary 3.12). In particular, the ring of algebraic integers in any algebraic number field satisfies power-substitution. This might lead one to expect that power-substitution is a property of Dedekind domains, or perhaps at least of principal ideal domains. This is false, however, for the polynomial ring $F[x]$ over any field $F$ of characteristic zero never satisfies power-substitution (Corollary 3.8). For noncommutative examples of power-substitution, we have any ring $R$ whose additive group has finite rank (Theorem 4.12).

We also have examples to show that power-substitution does not in general imply any of the stable range conditions. If $X$ is a compact Hausdorff space and $C(X)$ is the ring of all continuous real-valued functions on $X$, then we claim that $C(X)$ satisfies power-substitution. For if $a x+b=1$ in $C(X)$, then the functions $a, b$ are not both zero anywhere, whence $a^{2}+b^{2}>0$ everywhere. As a result, $a I+b\left(\begin{array}{rr}0 & 1 \\ -1 & 0\end{array}\right)$, which has determinant $a^{2}+b^{2}$, is a unit in $M_{2}(C(X))$.

As shown in [11, pp. 264-269], there is a category equivalence $\Gamma$ between the real vector bundles over $X$ and the finitely generated projective $C(X)$-modules. For a given positive integer $n$, let $\tau^{n}$ denote the tangent bundle to the $n$-sphere $S^{n}$. Then $\Gamma\left(\tau^{n}\right) \oplus C\left(S^{n}\right)$ is free of rank $n+1$, but $\Gamma\left(\tau^{n}\right)$ is not free unless $n=0,1,3,7$ [11, Example 1, p. 269]. Thus for $n>7, \quad C\left(S^{n}\right) \oplus C\left(S^{n}\right)^{n} \cong C\left(S^{n}\right) \oplus \Gamma\left(\tau^{n}\right)$ and $C\left(S^{n}\right)^{n} \not \equiv \Gamma\left(\tau^{n}\right)$, hence we see from Theorem 1.4 that $C\left(S^{n}\right)$ does not have $n$ in the stable range.

Now let $Y$ be a disjoint union of the spheres $S^{1}, S^{2}, S^{3}, \cdots$, and let $X$ be the Stone-Cech compactification of $Y$. Inasmuch as any bounded continuous map of $Y$ into $\mathbf{R}$ extends to a continuous map of $X$ into $\mathbf{R}$, we see that the various restriction maps $C(X) \rightarrow C\left(S^{n}\right)$ are surjective, i.e., $C\left(S^{n}\right)$ is isomorphic to a factor ring of $C(X)$. Since $C\left(S^{n}\right)$ does not have $n$ in the stable range for $n>7$, we see that $C(X)$ does not have any $n$ in the stable range, although $C(X)$ does satisfy power-substitution.

Theorem 2.1 and Corollary 2.2 can be used to show that certain abelian groups enjoy power-cancellation in the category of abelian groups. However, by taking a little care with the proof of Theorem 2.1, we can actually show that such abelian groups enjoy power-cancellation in the category of all groups. We must be careful with our notation in this situation. In order to avoid ambiguities, we use $\times{ }^{n} G$ to denote the direct product of $n$ copies of a group $G$. Given any subgroup $H$ of $G$, we identify $\times{ }^{n} H$ with its canonical image in $\times{ }^{n} G$. Also, we identify the factors in a direct product of groups with the appropriate normal subgroups of the product group. 
THEOREM 2.3. Let $A$ be an abelian group with the powersubstitution property. Given any group $M$ (not necessarily abelian) and decompositions $M=A_{1} \times B_{1}=A_{2} \times B_{2}$ with each $A_{i} \cong A$, there exist $a$ positive integer $n$ and a normal subgroup $C \subseteq \times{ }^{n} M$ such that $\times{ }^{n} M=$ $C \times\left(\times{ }^{n} B_{1}\right)=C \times\left(\times{ }^{n} B_{2}\right)$.

Proof. As in Theorem 2.1, given a group homomorphism $f: G \rightarrow H$, we use $f^{*}$ to denote the induced homomorphism $\times{ }^{n} G \rightarrow \times{ }^{n} H$.

Using the decomposition $M=A_{1} \times B_{1} \cong A \times B_{1}$, we obtain projections $p_{1}: M \rightarrow A, p_{2}: M \rightarrow B_{1}$ and injections $q_{1}: A \rightarrow M, q_{2}: B_{1} \rightarrow M$ such that $p_{1} q_{1}$ is the identity map on $A$, $\operatorname{ker} p_{1}=B_{1}$, and $\left[q_{1} p_{1}(x)\right]\left[q_{2} p_{2}(x)\right]=x$ for all $x \in M$. Using the decomposition $M=A_{2} \times B_{2} \cong A \times B_{2}$, we obtain a projection $f: M \rightarrow A$ and an injection $g: A \rightarrow M$ such that $f g$ is the identity map on $A$ and $\operatorname{ker} f=B_{2}$. Since $A$ is abelian, we see from these decompositions that $g(A)$ and $q_{1}(A)$ are contained in the center of $M$. For any $x \in A$, we thus have $q_{2} p_{2} g(x)=\left[q_{1} p_{1} g(x)\right]^{-1} g(x)$ in the center of $M$. Therefore $q_{2} p_{2} g$ maps $A$ into the center of $M$.

Clearly $\left[f q_{1} p_{1} g(x)\right]\left[f q_{2} p_{2} g(x)\right]=f g(x)=x$ for all $x \in A$. Since $f q_{1}, p_{1} g$, and $f q_{2} p_{2} g$ all belong to the ring $E=\operatorname{End}(A)$, this translates into additive notation as $\left(f q_{1}\right)\left(p_{1} g\right)+\left(f q_{2} p_{2} g\right)=1$ in $E$. Inasmuch as $A$ has the power-substitution property, $E$ satisfies right power-substitution, hence we may proceed as in Theorem 2.1 to find $n>0$ and an endomorphism $h$ of $\times^{n} A$ such that $f^{*} q_{1}^{*}+f^{*} q_{2}^{*} p_{2}^{*} g^{*} h$ is an automorphism of $\times^{n} A$.

Inasmuch as $q_{1}$ and $q_{2} p_{2} g$ map $A$ into the center of $M$, we see that $q_{1}^{*}$ and $q_{2}^{*} p_{2}^{*} g^{*}$ map $\times{ }^{n} A$ into the center of $\times{ }^{n} M$. As a result, the rule $k(x)=\left[q_{1}^{*}(x)\right]\left[q_{2}^{*} p_{2}^{*} g^{*} h(x)\right]$ defines a homomorphism $k$ of $\times^{n} A$ into the center of $\times^{n} M$, whence $C=k\left(\times^{n} A\right)$ is a normal subgroup of $\times^{n} M$. Since $p_{1}^{*} k=p_{1}^{*} q_{1}^{*}$ is the identity map on $\times^{n} A$, we thus obtain $\times{ }^{n} M=C \times\left(\operatorname{ker} p_{1}^{*}\right)=C \times\left(\times{ }^{n} B_{1}\right)$. We also know that $f^{*} k=$ $f^{*} q_{1}+f^{*} q_{2}^{*} p_{2}^{*} g^{*} h$ is an automorphism of $\times^{n} A$, from which we obtain $\times{ }^{n} M=C \times\left(\operatorname{ker} f^{*}\right)=C \times\left(\times{ }^{n} B_{2}\right)$.

COROllary 2.4. Let $A$ be an abelian group whose endomorphism ring has the right power-substitution property. If $B$ and $C$ are any groups (not necessarily abelian) such that $A \times B \cong A \times C$, then $\times{ }^{n} B \cong \times{ }^{n} C$ for some positive integer $n$.

Proposition 2.5. Let $K$ be a two-sided ideal of $R$.

(a) If $R$ satisfies right power-substitution, then so does $R / K$.

(b) If $K \subseteq J(R)$ and $R / K$ satisfies right power-substitution, then so does $R$.

Proof. (a) is trivial.

(b) If $a x+b=1$ in $R$, then $\overline{a x}+\bar{b}=1$ in $R / K$, hence there exist 
$n>0$ and $Q \in M_{n}(R)$ such that $\bar{a} I+\overline{b Q}$ is a unit in $M_{n}(R / K)$. Thus we have $a I+b Q \in M_{n}(R)$ which maps to a unit modulo $M_{n}(K) \subseteq$ $J\left(M_{n}(R)\right)$, whence $a I+b Q$ is a unit in $M_{n}(R)$.

Proposition 2.6. Let e be an idempotent in $R$. If $R$ satisfies right power-substitution, then so does eRe.

Proof. Suppose that $a, x, b \in e R e$ with $a x+b=e$. Then $(a+1-e)(x+1-e)+b=1$ in $R$, so there exist $n>0$ and $Q \in M_{n}(R)$ such that $(a+1-e) I+b Q$ is a unit in $M_{n}(R)$. Set $T=$ $[(a+1-e) I+b Q]^{-1}$, and note that $e T[(a+1-e) I+b Q]=e I$. Multiplying this equation on the right by $e$, we obtain $e T[a I+b Q e]=e I$; multiplying it on the right by $1-e$, we obtain $e T[(1-e) I+b Q(1-e)]=$ 0 . Combining these two results, we find that

$$
e T[a I+b Q]=e T[a I+b Q e]+e T[b Q(1-e)]=e I-e T(1-e) .
$$

As a result, we obtain

$$
\begin{aligned}
{[e T+(1-e) I][(a+1-e) I+b Q] } & =e T[a I+b Q]+e T(1-e)+(1-e) I \\
& =I,
\end{aligned}
$$

and consequently $e T+(1-e) I=[(a+1-e) I+b Q]^{-1}=T$.

We now observe that $T e=e T e$, whence

$$
e I=[(a+1-e) I+b Q] T e=[(a+1-e) I+b Q] e T e=[a I+b e Q e] e T e .
$$

On the other hand, we have seen above that $e T[a I+b Q e]=e I$, whence $e T e[a I+b e Q e]=e I$. Therefore we have $e Q e \in M_{n}(e R e)$ such that $a I+$ $b e Q e$ is a unit in $M_{n}(e R e)$.

The obvious complement to Proposition 2.6 would be to prove that if $R$ satisfies right power-substitution, then so does any $M_{n}(R)$. We do not know whether this is true. However, we do have partial results in this direction, which are needed later in the paper. They require the following lemma, which was suggested by R. B. Warfield, Jr.

Lemma 2.7. Let $k$ be a positive integer, and let $A$ be a right $R$-module such that $\operatorname{End}_{R}(A)$ has $k$ in the stable range. Given any right $R$-module decomposition

$$
M=A_{11} \oplus \cdots \oplus A_{1 n} \oplus B_{1}=A_{21} \oplus \cdots \oplus A_{2 n} \oplus B_{2}
$$

with $n \geqq k$ and all $A_{i j} \cong A$, there exist submodules $C, D, H \leqq M$ such that 


$$
M=C \oplus D \oplus H \oplus B_{1}=C \oplus D \oplus A_{21} \oplus \cdots A_{2, k-1} \oplus B_{2}
$$

and $D \cong A, D \oplus H \cong A^{k}$.

Proof. We first claim that there exist $C, J \leqq M$ such that

$$
M=C \oplus J \oplus B_{1}=C \oplus A_{21} \oplus \cdots \oplus A_{2 k} \oplus B_{2}
$$

and $J \cong A^{k}$. If $n=k$, take $C=0$ and $J=A_{11} \oplus \cdots \oplus A_{1 k}$. Now let $n>k$, and assume that the claim holds for $n-1$.

Using the case $n-1$ on the decomposition

$$
M=A_{11} \oplus \cdots \oplus A_{1, n-1} \oplus\left(A_{1 n} \oplus B_{1}\right)=A_{21} \oplus \cdots \oplus A_{2, n-1} \oplus\left(A_{2 n} \oplus B_{2}\right),
$$

we obtain $D, K \leqq M$ such that

$$
M=D \oplus K \oplus A_{1 n} \oplus B_{1}=D \oplus A_{21} \oplus \cdots \oplus A_{2 k} \oplus A_{2 n} \oplus B_{2}
$$

and $K \cong A^{k}$. Write $K=A_{11}^{\prime} \oplus \cdots \oplus A_{1 k}^{\prime}$ with each $A_{1 j}^{\prime} \cong A$. We now have a decomposition

$$
\begin{aligned}
M & =A_{11}^{\prime} \oplus \cdots \oplus A_{1 k}^{\prime} \oplus\left(A_{1 n} \oplus B_{1} \oplus D\right) \\
& =A_{2 n} \oplus\left(A_{21} \oplus \cdots \oplus A_{2 k} \oplus B_{2} \oplus D\right)
\end{aligned}
$$

with $A_{11}^{\prime} \cong \cdots \cong A_{1 k}^{\prime} \cong A_{2 n} \cong A$.

Since $\operatorname{End}_{R}(A)$ has $k$ in the stable range, Theorem 1.3 shows that there exist $E, L \leqq M$ such that

$$
M=E \oplus L \oplus A_{1 n} \oplus B_{1} \oplus D=E \oplus A_{21} \oplus \cdots \oplus A_{2 k} \oplus B_{2} \oplus D
$$

Now $E$ and $A_{2 n}$ are complements for $A_{21} \oplus \cdots \oplus A_{2 k} \oplus B_{2} \oplus D$ in $M$, whence $E \cong A_{2 n} \cong A$. Also, $E \oplus L$ and $K$ are complements for $A_{1 n} \oplus B_{1} \oplus D$ in $M$, hence

$$
L \oplus A_{1 n} \cong A \oplus L \cong E \oplus L \cong K \cong A^{k}
$$

Setting $C=D \oplus E$ and $J=L \oplus A_{\text {In }}$, we thus have

$$
M=C \oplus J \oplus B_{1}=C \oplus A_{21} \oplus \cdots \oplus A_{2 k} \oplus B_{2}
$$

with $J \cong A^{k}$. Thus the claim is proved.

By virtue of this claim, we obtain a decomposition $M=A_{11}^{\prime \prime} \oplus \cdots \oplus A_{1 k}^{\prime \prime} \oplus\left(B_{1} \oplus C\right)=A_{2 k} \oplus\left(A_{21} \oplus \cdots \oplus A_{2, k-1} \oplus B_{2} \oplus C\right)$ 
with $A_{11}^{\prime \prime} \cong \cdots \cong A_{1 k}^{\prime \prime} \cong A_{2 k} \cong A$. Using Theorem 1.3 again, there exist $D, H \leqq M$ such that

$$
M=D \oplus H \oplus B_{1} \oplus C=D \oplus A_{21} \oplus \cdots \oplus A_{2, k-1} \oplus B_{2} \oplus C .
$$

As above, we conclude that $D \cong A$ and $D \oplus H \cong A^{k}$.

Proposition 2.8. Let $k$ be a positive integer, and let $R$ be a ring which has $k$ in the stable range. If $M_{k}(R)$ satisfies right powersubstitution, then so does $M_{n}(R)$, for all $n$.

Proof. In view of Proposition 2.6, we need only consider the case $n>k$. Setting $A=R_{R}$, we are given that the module $A^{k}$ has the power-substitution property, and we must prove it for $A^{n}$.

Thus consider any right $R$-module decomposition $M=A_{1} \oplus B_{1}=$ $A_{2} \oplus B_{2}$ in which each $A_{i} \cong A^{n}$. Write each $A_{i}=A_{i 1} \oplus \cdots \oplus A_{\text {in }}$, where all $A_{i j} \cong A$. According to Lemma 2.7 , there exist $C, D, H \leqq M$ such that

$$
\begin{aligned}
M & =C \oplus D \oplus H \oplus B_{1}=C \oplus D \oplus A_{21} \oplus \cdots \oplus A_{2, k-1} \oplus B_{2} \\
& =(D \oplus H) \oplus\left(B_{1} \oplus C\right)=\left(A_{21} \oplus \cdots \oplus A_{2, k-1} \oplus D\right) \oplus\left(B_{2} \oplus C\right)
\end{aligned}
$$

and $D \cong A, D \oplus H \cong A^{k}$. Since $A^{k}$ has the power-substitution property, there must exist $s>0$ and $E \leqq M^{s}$ such that

$$
\begin{aligned}
M^{s} & =E \oplus\left(B_{1} \oplus C\right)^{s}=E \oplus\left(B_{2} \oplus C\right)^{s} \\
& =\left(E \oplus C^{s}\right) \oplus B_{1}^{s}=\left(E \oplus C^{s}\right) \oplus B_{2}^{s} .
\end{aligned}
$$

Therefore $A^{n}$ has power-substitution.

Proposition 2.9. Let $R$ be a commutative ring which has 2 in the stable range. If $R$ satisfies power-substitution, then so does $M_{n}(R)$, for all $n$.

Proof. With $A=R_{R}$, we must prove that the module $A^{n}$ has power-substitution, for all $n \geqq 2$.

Thus consider any $R$-module decomposition $M=A_{1} \oplus B_{1}=$ $A_{2} \oplus B_{2}$ in which each $A_{i} \cong A^{n}$. Write each $A_{i}=A_{i 1} \oplus \cdots \oplus A_{i n}$, where all $A_{i j} \cong A$. According to Lemma 2.7 , there exist $C, D, H \leqq M$ such that

$$
\begin{aligned}
M & =C \oplus D \oplus H \oplus B_{1}=C \oplus D \oplus A_{21} \oplus B_{2} \\
& =H \oplus\left(B_{1} \oplus C \oplus D\right)=A_{21} \oplus\left(B_{2} \oplus C \oplus D\right)
\end{aligned}
$$


and $D \cong A, D \oplus H \cong A^{2}$. Now $H$ is an $R$-module such that $R \oplus H \cong$ $R \oplus R$, whence $H \cong R=A[6$, Theorem, p. 76]. As a result, there must exist $k>0$ and $E \leqq M^{k}$ such that

$$
\begin{aligned}
M^{k} & =E \oplus\left(B_{1} \oplus C \oplus D\right)^{k}=E \oplus\left(B_{2} \oplus C \oplus D\right)^{k} \\
& =\left(E \oplus C^{k} \oplus D^{k}\right) \oplus B_{1}^{k}=\left(E \oplus C^{k} \oplus D^{k}\right) \oplus B_{2}^{k} .
\end{aligned}
$$

Therefore $A^{n}$ has power-substitution.

\section{Commutative examples.}

LEMMA 3.1. Let $R$ be a commutative ring. Given elements $a, b, a_{1}, \cdots, a_{n} \in R$, there exists a matrix $Q \in M_{n}(R)$ such that

$$
\operatorname{det}(a I+b Q)=a^{n}+a_{1} a^{n-1} b+a_{2} a^{n-2} b^{2}+\cdots+a_{n} b^{n} \text {. }
$$

Proof. Set

$$
Q=\left[\begin{array}{ccccc}
a_{1} & -a_{2} & a_{3} & \cdots & (-1)^{n+1} a_{n} \\
1 & 0 & 0 & \cdots & 0 \\
0 & 1 & 0 & \cdots & 0 \\
& & \vdots & & \\
0 & 0 & \cdots & 1 & 0
\end{array}\right]
$$

so that

$$
a I+b Q=\left[\begin{array}{ccccc}
a+a_{1} b & -a_{2} b & a_{3} b & \cdots & (-1)^{n+1} a_{n} b \\
b & a & 0 & \cdots & 0 \\
0 & b & a & \cdots & 0 \\
& & \vdots & & \\
0 & 0 & \cdots & b & a
\end{array}\right]
$$

The lemma clearly holds for $n=1,2$.

Now let $n>2$, and expand $\operatorname{det}(a I+b Q)$ by the right-hand column, which yields

$$
a_{n} b\left|\begin{array}{ccccc}
b & a & 0 & \cdots & 0 \\
0 & b & a & \cdots & 0 \\
& & \vdots & & \\
0 & 0 & \cdots & b & a \\
0 & 0 & \cdots & 0 & b
\end{array}\right|+a\left|\begin{array}{ccccc}
a+a_{1} b & -a_{2} b & a_{3} b & \cdots & (-1)^{n} a_{n-1} b \\
b & a & 0 & \cdots & 0 \\
0 & b & a & \cdots & 0 \\
& & \vdots & & \\
0 & 0 & \cdots & b & a
\end{array}\right|
$$


The first determinant is clearly $b^{n}$, while the second (by induction), is $a^{n-1}+a_{1} a^{n-2} b+\cdots+a_{n-1} b^{n-1}$, which yields the desired result.

Proposition 3.2. A commutative ring $R$ satisfies the powersubstitution property if and only if whenever $a x+b=1$ for some $a, x, b \in R$, there exist a positive integer $n$ and an element $y \in R$ such that $a^{n}+$ by is a unit in $R$.

Proof. First assume that $R$ satisfies power-substitution. Given $a x+b=1$ in $R$, there exist $n>0$ and $Q \in M_{n}(R)$ such that $a I+b Q$ is a unit in $M_{n}(R)$. Then $\operatorname{det}(a I+b Q)$ is a unit in $R$, and we observe that $\operatorname{det}(a I+b Q)=a^{n}+b y$ for some $y \in R$.

Conversely, let $a x+b=1$ in $R$ and assume that $a^{n}+b y$ is a unit in $R$, for some $n>0$ and some $y \in R$. Now

$$
a^{n}+b y=a^{n}+b y(a x+b)^{n-1}=a^{n}+a_{1} a^{n-1} b+a_{2} a^{n-2} b^{2}+\cdots+a_{n} b^{n}
$$

for suitable $a_{1}, \cdots, a_{n} \in R$. According to Lemma 3.1, there exists $Q \in M_{n}(R)$ for which $\operatorname{det}(a I+b Q)=a^{n}+b y$, whence $a I+b Q$ is a unit in $M_{n}(R)$.

COROLlaRy 3.3. Let $R$ be a commutative ring such that for all nonzero $b \in R$, the group of units of $R / b R$ is torsion. (In particular, this holds if $R / b R$ is finite for all nonzero $b \in R$.) Then $R$ satisfies powersubstitution.

Proof. Let $a x+b=1$ in $R$. If $b=0$, then $a^{1}+b 0$ is a unit, hence we may now assume that $b \neq 0$. Then $a$ maps to an element $\bar{a}$ in the group of units of $R / b R$. Since this group is torsion, we must have $\bar{a}^{n}=1$ for some $n>0$, hence $a^{n}+b y=1$ for some $y \in R$.

COROllary 3.4. All subrings of $\mathbf{Q}$ satisfy power-substitution.

COROLlaRY 3.5. If $F$ is a field which is an algebraic extension of a finite field, then the polynomial ring $F[x]$ satisfies power-substitution.

Proof. Since $F$ is algebraic over a finite field, it must be a directed union of finite subfields $F_{i}$, whence $F[x]$ is a directed union of the subrings $F_{i}[x]$. Each such $F_{i}[x]$ satisfies power-substitution by Corollary 3.3, from which we conclude that $F[x]$ satisfies power-substitution.

LEMMA 3.6. Let $R$ be a commutative ring, let $a, b, c, d \in R$, and set $f=x^{2}+c x+d$ in $R[x]$. If there exists $g \in R[x]$ such that $a+b x+f g$ is a unit in $R[x]$, then $b$ is nilpotent. 
Proof. Consider any prime ideal $P$ of $R$, and map everything into $(R / P)[x]$, where $\bar{a}+\bar{b} x+\overline{f g}$ is a unit. If $\bar{g} \neq 0$, then $\operatorname{deg}(\overline{f g}) \geqq 2$ and so $\operatorname{deg}(\bar{a}+\bar{b} x+\overline{f g}) \geqq 2$, which is impossible. Thus $\bar{g}=0$, so that $\bar{a}+\bar{b} x$ is a unit in $(R / P)[x]$. As a result, $\bar{b}=0$, i.e., $b \in P$.

Therefore $b$ lies in all prime ideals of $R$ and thus is nilpotent.

THEOREM 3.7. Let $R$ be a commutative ring. Then the polynomial ring $R[x]$ satisfies power-substitution if and only if

(a) $R$ has positive characteristic.

(b) The group of units of $R$ is torsion.

(c) $R$ has Krull dimension zero.

Proof. First assume that $R[x]$ satisfies power-substitution. Since $(1+x)(1-x)+x^{2}=1$, there exist $n>0$ and $t \in R[x]$ such that $(1+x)^{n}+x^{2} t$ is a unit in $R[x]$. Observing that $(1+x)^{n}+x^{2} t=$ $1+n x+x^{2} g$ for some $g \in R[x]$, we see from Lemma 3.6 that $n$ is nilpotent in $R$, i.e., $n^{k} R=0$ for some $k>0$. Thus $R$ has positive characteristic.

Next consider any unit $a \in R$, and observe that

$$
(a x+1-x)\left(a^{-1} x+1-x\right)+\left(x^{2}-x\right)\left(a+a^{-1}-2\right)=1 .
$$

Then there exist $n>0$ and $t \in R[x]$ such that $(a x+1-x)^{n}+\left(x^{2}-x\right) t$ is a unit in $R[x]$. Observing that $x$ and $1-x$ are orthogonal idempotents modulo $x^{2}-x$, we see that

$$
(a x+1-x)^{n}+\left(x^{2}-x\right) t=a^{n} x+1-x+\left(x^{2}-x\right) g
$$

for some $g \in R[x]$. According to Lemma 3.6, the element $c=a^{n}-1$ must be nilpotent, hence $c^{k}=0$ for some $k>0$. Inasmuch as $p R=0$ for some positive integer $p$, we compute that $(1+c)^{p k}=1+c^{p k}=1$, i.e., $a^{n p k}=1$. Thus the group of units of $R$ is torsion.

If $R$ is not zero-dimensional, then it has a prime ideal $P$ which is not maximal. Set $S=R / P$, and note from Proposition 2.5 that $S[x]$ has power-substitution. Now $S$ is a domain but not a field, hence there exists a nonzero element $a \in S$ which is not a unit. Obviously $a x+(1-a x)=$ 1 , whence there exist $n>0$ and $t \in S[x]$ such that $a^{n}+(1-a x) t$ is a unit in $S[x]$. Since $S$ is a domain, this can only happen for $t=0$. But then $a^{n}$ is a unit in $S$ and so $a$ is a unit, which is false. Therefore $R$ must be zero-dimensional.

Conversely, assume that (a), (b), (c) hold. Since $R$ is zerodimensional, $J(R)$ is nil and $R / J(R)$ is von Neumann regular. Inasmuch as the natural map from the group of units of $R$ to the group of units of $R / J(R)$ is surjective, we see that the group of units of $R / J(R)$ is 
torsion. Thus $R / J(R)$ satisfies conditions (a), (b), (c). Since $J(R)$ is nil, so is $J(R)[x]$, whence $J(R)[x] \subseteq J(R[x])$. According to Proposition 2.5 , it thus suffices to prove that $(R / J(R))[x]$ has power-substitution. Therefore we may assume, without loss of generality, that $R$ is von Neumann regular.

We claim that for any $M \in \operatorname{Spec}(R),(R / M)[x]$ satisfies powersubstitution. Since $R / M$ is a field of positive characteristic, its prime field is finite, hence by Corollary 3.5 it suffices to show that $R / M$ is algebraic over its prime field. Thus consider any nonzero $\bar{a} \in R / M$. Then $a R=e R$ for some idempotent $e \in R-M$, and we note that $1-e \in M$. Observing that $a+1-e$ is a unit in $R$, we see from (b) that $(a+1-e)^{n}=$ 1 for some $n>0$. Since $1-e \in M$, it follows that $\bar{a}^{n}=1$ in $R / M$, whence $\bar{a}$ is algebraic over the prime field of $R / M$. Thus the claim is proved.

Now let $a t+b=1$ in $R[x]$. For any $M \in \operatorname{Spec}(R)$, we have $\overline{a t}+\bar{b}=1$ in $(R / M)[x]$, hence there exist $n>0$ and $\bar{u}, \bar{v} \in(R / M)[x]$ such that $\left(\bar{a}^{n}+\overline{b u}\right) \bar{v}=1$, i.e., $\left(a^{n}+b u\right) v-1 \in M[x]$.

Let $X$ be the family of all clopen sets $W \subseteq \operatorname{Spec}(R)$ for which there exist $n>0$ and $u, v \in R[x]$ such that $\left(a^{n}+b u\right) v-1 \in M[x]$ for all $M \in W$. We have just seen above that given any $M \in \operatorname{Spec}(R)$, there exist $n>0$ and $u, v \in R[x]$ such that $\left(a^{n}+b u\right) v-1 \in M[x]$. Since there are only a finite number of coefficients from $M$ needed to express $\left(a^{n}+b u\right) v-1$, there must exist an idempotent $e \in M$ such that $\left(a^{n}+b u\right) v-1 \in e R[x]$. As a result,

$$
W=\left\{M^{\prime} \in \operatorname{Spec}(R) \mid e \in M^{\prime}\right\}=\left\{M^{\prime} \in \operatorname{Spec}(R) \mid 1-e \notin M^{\prime}\right\}
$$

is a member of $X$ which contains $M$. Thus $X$ covers $\operatorname{Spec}(R)$.

Inasmuch as $\operatorname{Spec}(R)$ is compact, it follows that we can cover it with pairwise disjoint clopen sets $W_{1}, \cdots, W_{k}$ from $X$. There exist orthogonal idempotents $e_{1}, \cdots, e_{k} \in R$ such that $e_{1}+\cdots+e_{k}=1$ and each $W_{i}=\left\{M \in \operatorname{Spec}(R) \mid e_{i} \notin M\right\}$.

For each $i=1, \cdots, k$, there exist $n(i)>0$ and $u_{i}, v_{i} \in R[x]$ such that $\left(a^{n(i)}+b u_{i}\right) v_{i}-1 \in M[x]$ for all $M \in W_{i}$. Set $n=n(1) n(2) \cdots n(k)$ and $s(i)=n / n(i)$ for each $i$. Since

$$
\left(a^{n(i)}+b u_{i}\right)^{s(i)} v_{i}^{s(i)}-1 \in M[x]
$$

for all $M \in W_{i}$, we see that there exist $w_{i}, z_{i} \in R[x]$ such that $\left(a^{n}+b w_{i}\right) z_{i}-1 \in M[x]$ for all $M \in W_{i}$.

Setting $w=e_{1} w_{1}+\cdots+e_{k} w_{k}$ and $z=e_{1} z_{1}+\cdots+e_{k} z_{k}$, we observe that

$$
e_{i}\left[\left(a^{n}+b w\right) z-1\right]=e_{i}\left[\left(a^{n}+b w_{i}\right) z_{i}-1\right] \in M[x]
$$

for all $M \in W_{i}$, whence $\left(a^{n}+b w\right) z-1 \in M[x]$ for all $M$ in any 
$W_{i}$. Since the $W_{i} \operatorname{cover} \operatorname{Spec}(R)$, and since $J(R)=0$, we conclude that $\left(a^{n}+b w\right) z-1=0$, i.e., $a^{n}+b w$ is a unit in $R[x]$.

Therefore $R[x]$ satisfies power-substitution.

COROllary 3.8. Let $F$ be a field. Then the polynomial ring $F[x]$ satisfies power-substitution if and only if $F$ is an algebraic extension of a finite field.

DEFINITION. If $R$ is a ring whose additive group is torsion-free of finite rank, then we refer to $R$ as a torsion-free finite rank $\mathbf{Z}$-algebra. In this section, we prove that any commutative, torsion-free, finite rank Z-algebra satisfies power-substitution. Although the commutativity hypothesis will be removed in the following section, we present the commutative case here because its proof is far simpler than the noncommutative case. We require the following lemma, which is also needed in the noncommutative case.

For use in the following proofs, we recall a few standard items from noncommutative ring theory. A ring $R$ is prime if the product of any two nonzero two-sided ideals of $R$ is nonzero; $R$ is semiprime if it has no nonzero nilpotent two-sided ideals. A module $A$ is finite-dimensional (in the sense of Goldie) provided $A$ contains no infinite direct sums of nonzero submodules. A right Goldie ring is a ring $R$ such that the right module $R_{R}$ is finite-dimensional and such that $R$ satisfies the $A C C$ on right annihilator ideals. We refer the reader to [3, Chapter 3] for the basic theory of finite-dimensional modules and Goldie rings.

LEMMA 3.9. Let $R$ be a semiprime, torsion-free, finite rank $\mathbf{Z}$ algebra. Then $R$ is right and left noetherian. For any right ideal $K$ of $R$, the following conditions are equivalent:

(a) $K$ is an essential right ideal of $R$.

(b) $K$ contains a non-zero-divisor of $R$.

(c) $k R \subseteq K$ for some positive integer $k$.

(d) $R / K$ is finite.

Proof. Since $R$ is torsion-free of finite rank, it contains no infinite direct sums of nonzero subgroups. Consequently, $R_{R}$ must be finitedimensional. Since $R$ is torsion-free, we see that the right annihilator of any subset of $R$ is a pure subgroup of $R$. Using finite rank once again, we see that $R$ has $A C C$ on pure subgroups, whence $R$ also has $A C C$ on right annihilators. Thus $R$ is a semiprime right Goldie ring.

Given any right ideal $K$ of $R$, we now obtain (a) $\Leftrightarrow(b)$ as a standard property of semiprime Goldie rings [3, Theorem 3.34]. The implications (d) $\Rightarrow$ (c) $\Rightarrow$ (b) are clear. Given (b), we see that $R$ is isomorphic to a 
subgroup of $K$. Inasmuch as $R$ is a torsion-free abelian group of finite rank, [5, Theorem 2.4] implies that $R / K$ is finite. Therefore (a)-(d) are equivalent.

Given any essential right ideal $K$ of $R$, we now know that $k R \subseteq K$ for some positive integer $k$, and that $R / k R$ is finite. As a result, $K$ must be finitely generated as a right ideal of $R$. Inasmuch as every right ideal of $R$ is a direct summand of an essential right ideal [3, Proposition 1.3], it follows that $R$ is right noetherian. By symmetry, $R$ is left noetherian as well.

THEOREM 3.10. If $R$ is a commutative, torsion-free, finite rank Z-algebra, then $R$ satisfies power-substitution.

Proof. Since $R$ is torsion-free, it embeds in the algebra $R \otimes \mathbf{Q}$. Now $R \otimes \mathbf{Q}$ is a commutative finite-dimensional $\mathbf{Q}$-algebra, hence $J(R \otimes \mathbf{Q})$ is nilpotent and $(R \otimes \mathbf{Q}) / J(R \otimes \mathbf{Q})$ has no nilpotent elements. As a result, we see that $R$ has a nilpotent ideal $N$ such that $R / N$ is a torsion-free $\mathbf{Z}$-algebra with no nilpotent elements. According to Proposition 2.5, it suffices to show that $R / N$ satisfies power-substitution. Thus we may assume, without loss of generality, that $R$ is a semiprime, commutative, torsion-free, finite rank $\mathbf{Z}$-algebra.

Suppose that $a x+b=1$ in $R$. Choose an ideal $K$ of $R$ such that $b R \oplus K$ is essential in $R$ [3, Proposition 1.3], and note that $b K=$ 0 . Note also that $\overline{a x}=1$ in $R /(b R \oplus K)$. According to Lemma 3.9, the ring $R /(b R \oplus K)$ is finite, whence its group of units is torsion. Thus $\bar{a}^{n}=1$ for some $n>0$, and consequently $a^{n}+b y+c=1$ for some $y \in R$, $c \in K$.

Since $a x+b=1, a^{n} x^{n}+b z=(a x+b)^{n}=1$ for a suitable $z \in R$, from which we obtain $\left(a^{n}+b y\right) x^{n}+b\left(z-y x^{n}\right)=1$. Multiplying this equation by the equation $\left(a^{n}+b y\right)+c=1$, and noting that $b c=0$, we conclude that $\left(a^{n}+b y\right) w=1$ for some $w \in R$, so that $a^{n}+b y$ is a unit.

Therefore $R$ satisfies power-substitution.

COROLlary 3.11. If $R$ is any commutative, torsion-free, algebraic Z-algebra, then $R$ satisfies power-substitution.

Proof. Since $R$ is a directed union of finitely generated subrings, it suffices to show that every finitely generated subring of $R$ satisfies power-substitution. Thus we may assume, without loss of generality, that $R$ is finitely generated, say $R=\mathbf{Z}\left[r_{1}, \cdots, r_{n}\right]$.

Since the $r_{i}$ are algebraic over $\mathbf{Z}$, there exists a positive integer $k$ such that $k r_{1}, \cdots, k r_{n}$ are integral over $\mathbf{Z}$. As a result, $\boldsymbol{S}=\mathbf{Z}\left[k r_{1}, \cdots, k r_{n}\right]$ is a finitely generated integral $\mathbf{Z}$-algebra, and thus is finitely generated as a $\mathbf{Z}$-module. Note that $R / S$ is a torsion group. Since $R$ is torsion-free, it 
follows that $R$ is a torsion-free finite rank $\mathbf{Z}$-algebra. By Theorem 3.10, $R$ satisfies power-substitution.

COROLlary 3.12. If $F$ is any algebraic field extension of $\mathbf{Q}$, then every subring of $F$ satisfies power-substitution.

COROLlaRy 3.13. If $R$ is any commutative ring which is integral over $\mathbf{Z}$, then $R$ satisfies power-substitution.

Proof. As in Corollary 3.11, we need only consider the case when $R$ is finitely generated (as a ring). Since $R$ is integral over $\mathbf{Z}$, we thus have that $R$ is a finitely generated $\mathbf{Z}$-module. In addition, Proposition 2.5 says that we need only show that $R / J(R)$ has power-substitution. Thus we may also assume that $J(R)=0$, so that $R$ is semiprime.

We claim that every finite ideal $I$ of $R$ is a direct summand of $R$. If $I$ is a minimal ideal, this follows from the semiprimeness of $R$ [7, Corollary, p. 63]. If $I \neq 0$, then $I$ must contain a minimal ideal $K$, and $R=K \oplus K^{\prime}$ for some $K^{\prime}$. Now $I=K \oplus\left(I \cap K^{\prime}\right)$, and by induction, $I \cap K^{\prime}$ is a direct summand of $R$. As a result, $I \cap K^{\prime}$ is a direct summand of $K^{\prime}$, hence we obtain $K^{\prime}=\left(I \cap K^{\prime}\right) \oplus K^{\prime \prime}$ for some $K^{\prime \prime}$, and consequently $R=I \oplus K^{\prime \prime}$.

Now let $T$ denote the torsion subgroup of $R$, which is a finite ideal of $R$ and thus is a direct summand of $R$. Since $R$ is commutative, this gives us a ring decomposition $R=S \times T$, where $S$ is a finitely generated free $\mathbf{Z}$-module. By Theorem 3.10, $S$ satisfies power-substitution. Since $T$ is artinian, it has 1 in the stable range [12, Lemma 11.8] and consequently satisfies power-substitution. Therefore $R$ satisfies power-substitution.

4. Finite rank $\mathbf{Z}$-algebras. Of the numerous definitions of "finite rank abelian group" in the literature, the following is best suited for our purposes, since it is clearly preserved by subgroups and homomorphic images.

Definition. An abelian group $A$ is said to have finite rank $[6, \mathrm{p}$. 49] provided there exists a positive integer $n$ such that every finitely generated subgroup of $A$ can be generated by $n$ elements. Note that if $A$ has finite rank, then $A / T(A)$ has finite rank and each of the primary components of $T(A)$ has $D C C$ on subgroups.

We refer to a ring $R$ whose additive group has finite rank as a finite rank $\mathbf{Z}$-algebra. The purpose of this section is to prove that every finite rank $\mathbf{Z}$-algebra satisfies power-substitution. Most of the difficulties occur in the torsion-free case, and the proof for this case involves a number of steps, covering matrix rings over noncommutative domains, orders over Dedekind domains, prime rings, and semiprime rings. 
LEMMA 4.1. Let $R$ be a semiprime, torsion-free, finite rank $\mathbf{Z}$ algebra. Let $a, x, b \in R$ such that $a x+b=1$. If $b$ is either zero or a nonzero-divisor, then there exist a positive integer $n$ and a matrix $Q \in M_{n}(R)$ such that $a I+b Q$ is a unit in $M_{n}(R)$.

Proof. If $b=0$, then $a x=1$. In view of Lemma $3.9, R$ is a semiprime right noetherian ring, whence $R$ is a subring of-a semisimple artinian ring. (This follows from Goldie's Theorem: [3, Theorem 3.35].) In this case $a x=1$ implies $x a=1$, so that $a^{1}+b 0$ is a unit in $R$.

Now assume that $b$ is a non-zero-divisor.

According to Lemma 3.9, there is a positive integer $k$ such that $k R \subseteq b R$, and $R / k R$ is finite. Now $R / k R$ is an artinian ring and so has 1 in the stable range [12, Lemma 11.8]. Inasmuch as $\overline{a x}+\bar{b}=1$ in $R / k R$, we thus obtain $y \in R$ such that $\bar{a}+\overline{b y}$ is a unit in $R / k R$. Since the group of units of $R / k R$ is finite, it follows that $(\bar{a}+\overline{b y})^{n}=1$ for some $n>0$. Since $k R \subseteq b R$, we thus obtain $(a+b y)^{n}+b z=1$ for some $z \in R$.

Set $c=a+b y$ and $d=b z$, so that $c^{n}+d=1$. Let $S$ denote the subring of $R$ generated by $c$ and $d$, which is commutaitve. Now

$$
1=c^{n}+d\left(c^{n}+d\right)^{n-1}=c^{n}+c_{1} c^{n-1} d+c_{2} c^{n-2} d^{2}+\cdots+c_{n} d^{n}
$$

for suitable $c_{1}, \cdots, c_{n} \in S$. As in Proposition 3.2, it follows that there exists $P \in M_{n}(S)$ such that $c I+d P$ is a unit in $M_{n}(S)$. As a result, we now have a matrix $Q=y I+z P$ in $M_{n}(R)$ such that $a I+b Q=c I+d P$ is a unit in $M_{n}(R)$.

Lemma 4.2. Let $R$ be a torsion-free finite rank $\mathbf{Z}$-algebra, and assume that $R$ is a domain (not necessarily commutative). Given any right $R$-module decomposition $M=A_{1} \oplus B_{1}=A_{2} \oplus B_{2}$ such that $R \oplus A_{1} \cong R^{2}$ and $A_{2} \cong R$, there exist a positive integer $n$ and a submodule $C \leqq M^{n}$ such that $M^{n}=C \oplus B_{1}^{n}=C \oplus B_{2}^{n}$.

Proof. Since every element of $R$ is either zero or a non-zerodivisor, we see from Lemma 4.1 that $R$ satisfies right power-substitution. Applying Corollary 2.2 to the relation $R \oplus A_{1} \cong R^{2}$, we thus find that $A_{1}^{s} \cong R^{s}$ for some $s>0$.

We now proceed in a manner close to the proof of Theorem 2.1. In order to keep the notation in line with that proof, we write $E$ for $\operatorname{End}_{R}\left(R_{R}\right)$.

Using the decomposition $M=A_{1} \oplus B_{1}$, we obtain projections $p_{1}: M \rightarrow A_{1}, p_{2}: M \rightarrow B_{1}$ and injections $q_{1}: A_{1} \rightarrow M, q_{2}: B_{1} \rightarrow M$. Using the decomposition $M=A_{2} \oplus B_{2} \cong R \oplus B_{2}$, we obtain a projection $f: M \rightarrow R$ and an injection $g: R \rightarrow M$. 
Now $1_{R}=f\left(q_{1} p_{1}+q_{2} p_{2}\right) g=\left(f q_{1}\right)\left(p_{1} g\right)+\left(f q_{2} p_{2} g\right)$. Setting $a=$ $f q_{1} \in \operatorname{Hom}_{R}\left(A_{1}, R\right), \quad x=p_{1} g \in \operatorname{Hom}_{R}\left(R, A_{1}\right)$, and $b=f q_{2} p_{2} g \in E$, we have $a x+b=1$ in $E$. Applying the $s$ th power functor, we obtain $a^{*} \in \operatorname{Hom}_{R}\left(A_{1}^{s}, R^{s}\right), x^{*} \in \operatorname{Hom}_{R}\left(R^{s}, A_{1}^{s}\right)$, and $b^{*} \in \operatorname{End}_{R}\left(R^{s}\right)=M_{s}(E)$ such that $a^{*} x^{*}+b^{*}=1$ in $M_{s}(E)$. Inasmuch as $E \cong R$ is a domain, $b$ is either zero or a non-zero-divisor in $E$, from which we see that $b^{*}$ is either zero or a non-zero-divisor in $M_{s}(E)$.

Choose an isomorphism $\phi: R^{s} \rightarrow A_{1}^{s}$. This gives us elements $a^{*} \phi$, $\phi^{-1} x^{*}, b^{*}$ in $M_{s}(E)$ such that $\left(a^{*} \phi\right)\left(\phi^{-1} x^{*}\right)+b^{*}=1$. Now $M_{s}(E)$ is a prime, torsion-free, finite rank $\mathbf{Z}$-algebra, hence Lemma 4.1 says that there exist $n>0$ and $Q \in M_{n}\left(M_{s}(E)\right)=M_{n s}(E)$ such that $a^{*} \phi I+b^{*} Q$ is a unit in $M_{n s}(E)$. Thus we now have a map $h=Q\left(\phi^{-1} I\right)$ in $\operatorname{Hom}_{R}\left(A_{1}^{n s}, R^{n s}\right)$ such that $a^{*}+b^{*} h$ is an isomorphism of $A_{1}^{n s}$ onto $R^{n s}$. Taking $C=\operatorname{ker}\left(q_{1}^{*}+q_{2}^{*} p_{2}^{*} g^{*} h\right)$, we conclude as in Theorem 2.1 that $M^{n s}=C \oplus B_{1}^{n s}=C \oplus B_{2}^{n s}$.

Proposition 4.3. Let $R$ be a torsion-free finite rank $\mathbf{Z}$-algebra. If $R$ is Morita-equivalent to a domain $S$ (not necessarily commutative), then $R$ satisfies right power-substitution.

Proof. Since $S$ is isomorphic to the endomorphism ring of a finitely generated projective $R$-module, we see that $S$ is torsion-free and finite rank over $\mathbf{Z}$.

On the other hand, there exist a positive integer $n$ and an idempotent $e \in M_{n}(S)$ such that $R \cong e M_{n}(S) e$. By Proposition 2.6, it suffices to show that $M_{n}(S)$ has right power-substitution, or equivalently, that the module $\left(S_{S}\right)^{n}$ has the power-substitution property. For $n=1$, this follows from Lemma 4.1, hence we need only consider the case $n \geqq 2$.

Now let $M=A_{1} \oplus B_{1}=A_{2} \oplus B_{2}$ be any right $S$-module decomposition such that each $A_{i} \cong S^{n}$. Write each $A_{i}=A_{i 1} \oplus \cdots \oplus A_{i n}$ with all $A_{i j} \cong S$. According to [14, Theorem 9] (with the help of Lemma 3.9), $S$ has 2 in the stable range. As a result, Lemma 2.7 shows that there exist $C, D, H \leqq M$ such that

$$
\begin{aligned}
M & =C \oplus D \oplus H \oplus B_{1}=C \oplus D \oplus A_{21} \oplus B_{2} \\
& =H \oplus\left(C \oplus D \oplus B_{1}\right)=A_{21} \oplus\left(C \oplus D \oplus B_{2}\right)
\end{aligned}
$$

and $D \cong S, \quad D \oplus H \cong S^{2}$. Thus $S \oplus H \cong S^{2}$ and $A_{21} \cong S$, whence Lemma 4.2 says that there exist $k>0$ and $F \leqq M^{k}$ such that

$$
\begin{aligned}
M^{k} & =F \oplus\left(C \oplus D \oplus B_{1}\right)^{k}=F \oplus\left(C \oplus D \oplus B_{2}\right)^{k} \\
& =\left(F \oplus C^{k} \oplus D^{k}\right) \oplus B_{1}^{k}=\left(F \oplus C^{k} \oplus D^{k}\right) \oplus B_{2}^{k} .
\end{aligned}
$$

Therefore $S^{n}$ has the power-substitution property, as required. 
LEMMA 4.4. Let $T$ be a ring satisfying right power-substitution, let $K$ be a two-sided ideal of $T$, and let $R$ be a subring of $T$ which contains $K$. If $R / K$ has 1 in the stable range (in particular, if $R / K$ is right artinian), then $R$ satisfies right power-substitution.

Proof. Let $a x+b=1$ in $R$. Since $R / K$ has 1 in the stable range, there exists $z \in R$ such that $\bar{a}+\overline{b z}$ is a unit in $R / K$. Now $(a+b z) x+$ $b(1-z x)=1$, and it suffices to find $n>0$ and $Q \in M_{n}(R)$ such that $(a+b z) I+b(1-z x) Q$ is a unit. Thus we may assume, without loss of generality, that $\bar{a}$ is a unit in $R / K$.

Now $a w+k=1$ for some $w \in R, k \in K$, and we note that $b k \in K$. Observing that $k=a x k+b k$, we obtain $a(w+x k)+b k=1$. Since it suffices to make $a I+b k Q$ a unit in some $M_{n}(R)$, we may now assume also that $b \in K$.

Since $a x+b=1, a x$ must commute with $b$, and consequently $1=(a x+b)^{2}=a x^{\prime}+b^{2}$ for some $x^{\prime} \in R$. Inasmuch as $T$ satisfies right power-substitution, there exist $n>0$ and $P \in M_{n}(T)$ such that $a I+b^{2} P$ is a unit in $M_{n}(T)$. Note that $Q=b P$ lies in $M_{n}(R)$, because $b T \subseteq K \subseteq R$. We now have $A=a I+b Q$ in $M_{n}(R)$ which has an inverse $B$ in $M_{n}(T)$. As a result, $\bar{B}$ is an inverse for $\bar{A}$ in $M_{n}(T / K)$. Since $\bar{a}$ is a unit in $R / K, \bar{A}=\bar{a} I$ also has an inverse in $M_{n}(R / K)$, from which we conclude that $\bar{B} \in M_{n}(R / K)$. Therefore $B \in M_{n}(R)$, whence $a I+b Q$ is a unit in $M_{n}(R)$, as desired.

Definition. A separable algebra over a field $F$ is a finitedimensional semisimple algebra $R$ such that the center of each simple component of $R$ is a separable field extension of $F$. (In particular, every finite-dimensional semisimple algebra over a field of characteristic zero is separable.) Equivalently, a finite-dimensional algebra $R$ over a field $F$ is separable if and only if $R$ is projective as a module over the algebra $R \otimes_{F} R^{o p}[9$, Theorem 7.20].

Definition. Let $S$ be a Dedekind domain with quotient field $F$, and let $Q$ be a (finite-dimensional) separable $F$-algebra. An $S$-order in $Q$ is any $S$-subalgebra $R$ of $Q$ such that $F R=Q$ and $R$ is finitely generated as an $S$-module. A maximal $S$-order is one which is maximal with respect to inclusion among the $S$-orders in $Q$. Every $S$-order is contained in a maximal $S$-order [9, Corollary 10.4].

Proposition 4.5. Let $S$ be a Dedekind domain which is a torsionfree finite rank $\mathbf{Z}$-algebra, let $Q$ be a finite-dimensional simple algebra over the quotient field of $S$, and let $R$ be an $S$-order in $Q$. Then $R$ satisfies right power-substitution. 
Proof. Note that the quotient field $F$ of $S$ has characteristic zero, so that $Q$ is a separable algebra over $F$. Also, $F$ is finite-dimensional over $\mathbf{Q}$, hence so is $Q$. As a result, we see that every $S$-order in $Q$ is a prime, torsion-free, finite rank $\mathbf{Z}$-algebra.

We know that $R$ must be contained in a maximal $S$-order $T$ in $Q$. The maximality of $T$ implies that $T$ is Morita-equivalent to a domain, as follows from [10, Chapter IV, Theorem 5.5]. By Proposition 4.3, $T$ has right power-substitution.

Choose generators $t_{1}, \cdots, t_{n}$ for $T$ as an $S$-module. There exist elements $r_{1}, \cdots, r_{n} \in R$ and $s \in S$ such that each $t_{i}=r_{i} / s$, from which we see that $s T \subseteq R$. Note that $s T$ is a two-sided ideal of $T$, and also an essential right ideal of $R$. According to Lemma 3.9, $R / s T$ is finite, hence artinian. By Lemma 4.4, we conclude that $R$ satisfies right powersubstitution.

Definition. Let $R$ be a subring of a ring $Q$. Then $R$ is called a right order in $Q$ provided every non-zero-divisor of $R$ is invertible in $Q$ and every element of $Q$ can be expressed in the form $a b^{-1}$ for suitable $a, b \in R, b$ a non-zero-divisor. Any $S$-order as defined above is also a right order in this sense.

Lemma 4.6. Let $Q$ be a finite-dimensional simple Q-algebra, and let $R$ be a right order in $Q$. Then there exists a noetherian domain $S$, contained in the centers of $R$ and $Q$, such that $R$ is a finitely generated $S$-module.

Proof. Note that $R$ is a prime, torsion-free, finite rank $\mathbf{Z}$-algebra. Given any $x \in Q$, we have $x=a b^{-1}$ for some $a \in R$ and some non-zerodivisor $b \in R$. According to Lemma 3.9, $k R \subseteq b R$ for some positive integer $k$, whence $k x \in R$. As a result, we find that $\mathbf{Q} R=Q$. According to [8, Theorem, p. 242], there exist a field $F$ contained in the center of $Q$, a basis $q_{1}, \cdots, q_{k}$ for $Q$ over $F$, and a nonzero integer $m$ such that

$$
m R \subseteq(R \cap F) q_{1}+\cdots+(R \cap F) q_{k} .
$$

Set $S=R \cap F$, which is a domain contained in the centers of $R$ and $Q$. Since $S$ is a torsion-free finite rank $\mathbf{Z}$-algebra, Lemma 3.9 shows that $S$ is noetherian. As a result, we see that $m R$ is a finitely generated $S$-module, hence so is $R$.

LEMMA 4.7. Let $F$ be a finite-dimensional field extension of $\mathbf{Q}$, let $\mathbf{S}$ be a domain with quotient field $F$, and let $T$ be the integral closure of $S$ in $F$. Then $T$ is a Dedekind domain, and $T$ is a finitely generated $S$-module. 
Proof. If $V$ is the integral closure of $Z$ in $F$, then $V$ is a Dedekind domain with quotient field $F$, hence any ring between $V$ and $F$ is also a Dedekind domain. In particular, $V \subseteq T \subseteq F$, whence $T$ is a Dedekind domain.

Now $V$ is finitely generated as a $\mathbf{Z}$-module, and so $S V$ is finitely generated as an $S$-module. Inasmuch as $S V$ contains $V$, it is a Dedekind domain with quotient field $F$, and so is integrally closed in $F$. In addition, $S \subseteq S V \subseteq T$, hence we find that $S V=T$. Thereforc $T$ is finitely generated as an $S$-module.

Proposition 4.8. If $R$ is a prime, torsion-free, finite rank $\mathbf{Z}$-algebra, then $R$ satisfies right power-substitution.

Proof. Since $R$ is right noetherian by Lemma 3.9, it must be a right order in a simple artinian ring $Q$ [3, Corollary 3.36]. Every nonzero integer is a non-zero-divisor in $R$ and so is invertible in $Q$, whence $Q$ is a $\mathbf{Q}$-algebra. As in Lemma 4.6, we obtain $\mathbf{Q} R=Q$, from which we see that $Q$ is a finite-dimensional $\mathbf{Q}$-algebra. Now Lemma 4.6 shows that there exists a noetherian domain $S$, contained in the centers of $R$ and $Q$, such that $R$ is a finitely generated $S$-module.

Let $F$ denote the quotient field of $S$, which we may view as a subfield of the center of $Q$, and let $T$ denote the integral closure of $S$ in $F$. According to Lemma 4.7, $T$ is a Dedekind domain and also a finitely generated $S$-module.

Note that $Q$ is a finite-dimensional simple $F$-algebra, and that $T R$ is a $T$-subalgebra of $Q$. Since $R$ is a finitely generated $S$-module, $T R$ is a finitely generated $T$-module. Also, $\mathbf{Q} R=Q$ implies that $F T R=Q$, whence $T R$ is a $T$-order in $Q$. Thus, according to Proposition 4.5, TR satisfies right power-substitution.

Inasmuch as $T$ is a finitely generated $S$-module, there must be a nonzero element $s \in S$ such that $s T \subseteq S$. As a result, $s T R$ is a two-sided ideal of $T R$ which is contained in $R$. Observing that $s T R$ is an essential right ideal of $R$, we see from Lemma 3.9 that $R / s T R$ is finite. Therefore $R$ satisfies right power-substitution, by Lemma 4.4.

Proposition 4.9. If $R$ is a semiprime, torsion-free, finite rank Z-algebra, then $R$ satisfies right power-substitution.

Proof. Since $R$ is right noetherian by Lemma 3.9, it must be a right order in a semisimple artinian ring $Q$ [3, Theorem 3.35]. Write $Q=$ $Q_{1} \times \cdots \times Q_{n}$, with each $Q_{i}$ simple. If $R_{i}$ denotes the image of the projection $R \rightarrow Q \rightarrow Q_{i}$, then $R_{i}$ is a right order in $Q_{i}$ and so is a prime ring. Let $T=R_{1} \times \cdots \times R_{n}$, so that $R \subseteq T \subseteq Q$. 
As in Proposition 4.8, $Q$ is a finite-dimensional Q-algebra. Then $T$ is a torsion-free finite rank $\mathbf{Z}$-algebra, and consequently Proposition 4.8 shows that $T$ satisfies right power-substitution.

Setting $K_{i}=R \cap Q_{i}$, we check that $K_{i}$ is a two-sided ideal of $R_{i}$, whence $K=K_{1} \times \cdots \times K_{n}$ is a two-sided ideal of $T$. In addition, $K$ is an essential right ideal of $R$, hence $R / K$ is finite by Lemma 3.9. Thus Lemma 4.4 shows that $R$ satisfies right power-substitution.

Proposition 4.10. If $R$ is a torsion-free finite rank $\mathbf{Z}$-algebra, then $R$ satisfies right power-substitution.

Proof. Since $R$ is torsion-free, we may identify it with its canonical image in $R \otimes \mathbf{Q}$. Now $R \otimes \mathbf{Q}$ is a finite-dimensional $\mathbf{Q}$-algebra, hence $J(R \otimes \mathbf{Q})$ is nilpotent and $(R \otimes \mathbf{Q}) / J(R \otimes \mathbf{Q})$ is semisimple artinian. Consequently, we see that $N=R \cap J(R \otimes \mathbf{Q})$ is a nilpotent two-sided ideal of $R$, and $R / N$ is a right order in $(R \otimes \mathbf{Q}) / J(R \otimes \mathbf{Q})$. Thus $R / N$ is a semiprime, torsion-free, finite rank Z-algebra, whence Proposition 4.9 shows that $R / N$ satisfies right power-substitution. According to Proposition $2.5, R$ must satisfy right power-substitution.

LEMMA 4.11. Let $S$ be a ring such that $M_{n}(S)$ satisfies right powersubstitution for all $n$, let $K$ be a two-sided ideal of $S$, and let $R$ be a subring of $S$ which contains $K$. If $R / K$ satisfies right power-substitution, then so does $R$.

Proof. Given $a x+b=1$ in $R$, there exist $n>0$ and $Q \in M_{n}(R)$ such that $\bar{a} I_{n}+\overrightarrow{b Q}$ is a unit in $M_{n}(R) / M_{n}(K)$. Set $S^{\prime}=M_{n}(S), K^{\prime}=$ $M_{n}(K), \quad R^{\prime}=M_{n}(R), \quad a^{\prime}=a I_{n}+b Q, \quad x^{\prime}=x I_{n}, \quad$ and $\quad b^{\prime}=b\left(I_{n}-Q x^{\prime}\right)$. Then $a^{\prime} x^{\prime}+b^{\prime}=1$ in $R^{\prime}$ and $\overline{a^{\prime}}$ is a unit in $R^{\prime} / K^{\prime}$.

Proceeding as in Lemma 4.4, there exist $k>0$ and $P \in M_{k}\left(R^{\prime}\right)$ such that $a^{\prime} I_{k}+b^{\prime} P$ is a unit in $M_{k}\left(R^{\prime}\right)$. As a result, we have $Q I_{k}+\left(I_{n}-Q x^{\prime}\right) P$ in $M_{k n}(R)$ such that $a I_{k n}+b\left[Q I_{k}+\left(I_{n}-Q x^{\prime}\right) P\right]$ is a unit in $M_{k n}(R)$.

THEOREM 4.12. If $R$ is any finite rank $\mathbf{Z}$-algebra, then $R$ satisfies the right and left power-substitution properties.

Proof. By symmetry, we need only check right power-substitution. By Proposition 2.5, it suffices to prove that $R / J(R)$ has right powersubstitution. Thus we may assume, without loss of generality, that $J(R)=0$, so that $R$ is semiprime.

Let $T$ denote the torsion subgroup of $R$, which is a two-sided ideal of $R$. For each prime integer $p$, let $T_{p}$ denote the $p$-primary component of $T$, which also is a two-sided ideal of $R$. Since $R$ has finite rank over $\mathbf{Z}$, $T_{p}$ must have $D C C$ on subgroups and hence also on right $R$-submodules. 
Because $R$ is semiprime, all minimal right ideals of $R$ are direct sumands of $R_{R}$, from which we infer (as in Corollary 3.13) that $T_{p}=e_{p} R$ for some idempotent $e_{p}$. Using semiprimeness again, it follows that $e_{p}$ is central. Note that the idempotents $e_{p}$ are pairwise orthogonal, and that $T=$ $\oplus e_{p} R$.

For any positive integer $n, M_{n}\left(\Pi e_{p} R\right) \cong \Pi M_{n}\left(e_{p} R\right)$ is a direct product of artinian rings. Since artinian rings have 1 in the stable range [12, Lemma 11.8], so does $M_{n}\left(\Pi e_{p} R\right)$, whence $M_{n}\left(\Pi e_{p} R\right)$ satisfies right power-substitution. In addition, $M_{n}(R / T)$ is a torsion-free finite rank Z-algebra, hence Proposition 4.10 shows that $M_{n}(R / T)$ satisfies right power-substitution. Setting $S=(R / T) \times\left(\Pi e_{p} R\right)$, we thus see that $M_{n}(S)$ satisfies right power-substitution for all $n$.

Observing that $T \cap\left[\cap\left(1-e_{p}\right) R\right]=0$, we obtain an injective ring map $\phi: R \rightarrow S$. Note that $\phi(T)=\{0\} \times\left(\bigoplus e_{p} R\right)$, which is a two-sided ideal of $S$. Inasmuch as $\phi(R) / \phi(T) \cong R / T$ satisfies right powersubstitution, so does $\phi(R) \cong R$, by Lemma 4.11 .

COROllary 4.13. If $R$ is any direct limit of finite rank $\mathbf{Z}$-algebras, then $R$ satisfies the right and left power-substitution properties.

\section{Applications.}

THEOREM 5.1. Let $A$ be a torsion-free abelian group of finite rank, and let $B, C$ be arbitrary groups (not necessarily abelian). If $A \times B \cong$ $A \times C$, then $\times{ }^{n} B \cong \times{ }^{n} C$ for some positive integer $n$.

Proof. Since the endomorphism ring of $A$ is a torsion-free finite rank Z-algebra, we may apply Theorem 4.12 and Corollary 2.4.

The case $A=\mathbf{Z}$ of Theorem 5.1 was proved by Hirshon in [4, Theorem 1]. A restricted version of this case was also proved by Warfield in [13, Theorem 2.1]. In addition, the case of Theorem 5.1 where $A, B, C$ are all torsion-free abelian of finite rank has been proved by Warfield (unpublished), using entirely different methods.

Lemma 5.2. Let $\mathscr{F}$ denote the class of all direct limits of finite rank Z-algebras.

(a) $\mathscr{F}$ is closed under subrings and factor rings.

(b) If $R \in \mathscr{F}$ and $A$ is a finitely generated right $R$-module, then $\operatorname{End}_{R}(A) \in \mathscr{F}$.

(c) If $R \in \mathscr{F}$ and $R \rightarrow S$ is a ring map such that $S$ is finitely generated as a right $R$-module, then $S \in \mathscr{F}$.

(d) If $R$ is a commutative ring which is either integral over $\mathbf{Z}$ or torsion-free and algebraic over $\mathbf{Z}$, then $R \in \mathscr{F}$. 
Proof. Note that a ring $R$ belongs to $\mathscr{F}$ if and only if every finitely generated subring of $R$ is a finite rank $\mathbf{Z}$-algebra.

(a) is clear.

(b) Choose a finitely generated free right $R$-module $F$ such that $A$ is isomorphic to a factor module of $F$. Then $\operatorname{End}_{R}(F)$ contains a subring $S$ such that $\operatorname{End}_{R}(A)$ is isomorphic to a factor ring of $S$. Since $\operatorname{End}_{R}(F)$ is isomorphic to a direct limit of full matrix rings over finitely generated subrings of $R$, we see that $\operatorname{End}_{R}(F) \in \mathscr{F}$, where $S \in \mathscr{F}$, and consequently $\operatorname{End}_{R}(A) \in \mathscr{F}$.

(c) According to (b), End $\operatorname{En}_{R}\left(S_{R}\right) \in \mathscr{F}$, whence $S \in \mathscr{F}$.

(d) If $R$ is integral over $\mathbf{Z}$, then every finitely generated subring of $R$ is also finitely generated as a $\mathbf{Z}$-module. If $R$ is torsion-free and algebraic over $\mathbf{Z}$, then (as in Corollary 3.11), every finitely generated subring of $R$ is a torsion-free finite rank $\mathbf{Z}$-algebra.

THEOREM 5.3. Let $S$ be a commutative ring which is either integral over $\mathbf{Z}$ or torsion-free and algebraic over $\mathbf{Z}$, let $T$ be an $S$-algebra which is finitely generated as an $S$-module, and let $R$ be any subring of $T$. Let $A$ be a finitely generated right $R$-module, and let $B, C$ be any right $R$ modules. If $A \oplus B \cong A \oplus C$, then $B^{n} \cong C^{n}$ for some positive integer $n$.

Proof. According to Lemma 5.2, $\operatorname{End}_{R}(A)$ is a direct limit of finite rank Z-algebras. Now apply Corollaries 4.13 and 2.2.

For example, Theorem 5.3 applies when $R$ is a subring of the group algebra $F[G]$ of a finite group $G$ over a field $F$ which is algebraic over $\mathbf{Q}$.

Definition. A right $R$-module $A$ is nonsingular provided $x I \neq 0$ for all nonzero $x \in A$ and all essential right ideals $I$ of $R$. A right nonsingular ring is a ring $R$ for which the right module $R_{R}$ is nonsingular. We refer the reader to $[3$, Chapter 1$]$ for an exposition of these concepts.

THEOREM 5.4. Let $R$ be a right nonsingular ring whose maximal right quotient ring $Q$ is a direct limit of finite rank $\mathrm{Z}$-algebras. Let $A$ be $a$ finite-dimensional nonsingular right $R$-module, and let $B, C$ be any right $R$-modules. If $A \oplus B \cong A \oplus C$, then $B^{n} \cong C^{n}$ for some positive integer $n$.

Proof. Since $A$ is nonsingular and finite-dimensional, its injective hull $E(A)$ is a finitely generated right $Q$-module [3, Theorem 3.16]. Also, it follows from the nonsingularity of $A$ that $\operatorname{End}_{R}(A)$ is naturally isomorphic to a subring of $\operatorname{End}_{O}(E(A))$. Consequently, we see from Lemma 5.2 that $\operatorname{End}_{R}(A)$ is a direct limit of finite rank $\mathbf{Z}$-algebras. Now apply Corollaries 4.13 and 2.2 . 
6. Problems. A. Is the power-substitution property (for rings) left-right symmetric?

B. Is it Morita-invariant?

C. Does Theorem 5.1 hold for finite rank abelian groups which are not necessarily torsion-free? In particular, does the endomorphism ring of such a group satisfy power-substitution? (The answer to both questions is yes in case the torsion subgroup of the group is a direct summand.)

D. Does a noncommutative algebraic $\mathbf{Q}$-algebra satisfy powersubstitution?

E. Presumably Theorem 4.12 can be generalized to finite rank algebras over some domains other than $\mathbf{Z}$. Perhaps it would work for a Dedekind domain $S$ such for all nonzero $b \in S$, the group of units of $S / b S$ is torsion.

\section{REFERENCES}

1. E. G. Evans, Jr., Krull-Schmidt and cancellation over local rings, Pacific J. Math., 46 (1973), 115-121.

2. L. Fuchs, On a substitution property for modules, Monatshefte für Math., 75 (1971), 198-204.

3. K. R. Goodearl, Ring Theory: Nonsingular Rings and Modules, New York (1976) Marcel Dekker (Pure and Applied Math. Series, Vol. 33).

4. R. Hirshon, The cancellation of an infinite cyclic group in direct products, Arch. der Math.,26 (1975), 134-138.

5. B. Jónsson, On direct decomposition of torsion-free abelian groups, Math. Scand., 7 (1959), 361-371.

6. I. Kaplansky, Infinite Abelian Groups (revised edition), Ann Arbor (1969), Univ. of Michigan Press.

7. J. Lambek, Lectures on Rings and Modules, Waltham, Mass. (1966) Blaisdell.

8. R. S. Pierce, Subrings of simple algebras, Michigan Math. J., 7 (1960), 241-243.

9. I. Reiner, Maximal Orders, New York (1975), Academic Press.

10. K. W. Roggenkamp and V. Huber-Dyson, Lattices over Orders I, Springer Lecture Notes No. 115, Berlin (1970), Springer-Verlag.

11. R. G. Swan, Vector bundles and projective modules, Trans. Amer. Math. Soc., 105 (1962), 264-277.

12. - Algebraic K-Theory, Springer Lecture Notes No. 76, Berlin (1968), Springer-Verlag.

13. R. B. Warfield, Jr., Genus and cancellation for groups with finite commutator subgroup, J. Pure Applied Algebra, 6 (1975), 125-132.

14. - Notes on cancellation, stable range, and related topics, Univ. of Washington, (August 1975).

Received November 14, 1975 and in reyised form January 26, 1976.

UNIVERSITY OF UTAH 



\section{PACIFIC JOURNAL OF MATHEMATICS}

\section{EDITORS}

RICHARD ARENS (Managing Editor)

University of California

Los Angeles, California 90024

\author{
R. A. Beaumont \\ University of Washington \\ Seattle, Washington 98105
}

\section{J. DugunduI}

Department of Mathematics University of Southern California Los Angeles, California 90007

D. Gilbarg and J. Milgram

Stanford University

Stanford, California 94305

\section{ASSOCIATE EDITORS}

E. F. BECKENBACH
B. H. NeumanN

F. Wolp
K. YoshidA

\section{SUPPORTING INSTITUTIONS}

UNIVERSITY OF BRITISH COLUMBIA

CALIFORNIA INSTITUTE OF TECHNOLOGY

UNIVERSITY OF CALIFORNIA

MONTANA STATE UNIVERSITY

UNIVERSITY OF NEVADA

NEW MEXICO STATE UNIVERSITY

OREGON STATE UNIVERSITY

UNIVERSITY OF OREGON

OSAKA UNIVERSITY

\author{
UNIVERSITY OF SOUTHERN CALIFORNIA \\ STANFORD UNIVERSITY \\ UNIVERSITY OF HAWAII \\ UNIVERSITY OF TOKYO \\ UNIVERSITY OF UTAH \\ WASHINGTON STATE UNIVERSITY \\ UNIVERSITY OF WASHINGTON \\ AMERICAN MATHEMATICAL SOCIETY
}

The Supporting Institutions listed above contribute to the cost of publication of this Journal, but they are not owners or publishers and have no responsibility for its contents or policies.

Mathematical papers intended for publication in the Pacific Journal of Mathematics should be in typed form or offset-reproduced (not dittoed). double spaced with large margins. Underline Greek letters in red, German in green, and script in blue. The first paragraph or two must be capable of being used separately as a synopsis of the entire paper. Items of the biblography should not be cited there unless absolutely necessary, in which case they must be identified by author and Journal, rather than by item number. Manuscripts, in duplicate, may be sent to any one of the four editors. Please classify according to the scheme of Math. Reviews, Index to Vol. 39. All other communications should be addressed to the managing editor, or Elaine Barth, University of California, Los Angeles, California, 90024.

100 reprints are provided free for each article, only if page charges have been substantially paid. Additional copies may be obtained at cost in multiples of 50 .

The Pacific Journal of Mathematics is issued monthly as of January 1966. Regular subscription rate: $\$ 72.00$ a year (6 Vols., 12 issues). Special rate: $\$ 36.00$ a year to individual members of supporting institutions.

Subscriptions, orders for back numbers, and changes of address should be sent to Pacific Journal of Mathematics, 103 Highland Boulevard, Berkeley, California, 94708.

PUBLISHED BY PACIFIC JOURNAL OF MATHEMATICS, A NON-PROFIT CORPORATION Printed at Jerusalem Academic Press, POB 2390, Jerusalem, Israel.

Copyright (C) 1976 Pacific Journal of Mathematics All Rights Reserved 


\section{Pacific Journal of Mathematics}

\section{Vol. 64, No. $2 \quad$ June, 1976}

Richard Fairbanks Arnold and A. P. Morse, Plus and times............. 297

Edwin Ogilvie Buchman and F. A. Valentine, External visibility ......... 333

R. A. Czerwinski, Bonded quadratic division algebras.............. 341

William Richard Emerson, Averaging strongly subadditive set functions in unimodular amenable groups. II .........................

Lynn Harry Erbe, Existence of oscillatory solutions and asymptotic behavior for a class of third order linear differential equations ............. 369

Kenneth R. Goodearl, Power-cancellation of groups and modules........ 387

J. C. Hankins and Roy Martin Rakestraw, The extremal structure of locally compact convex sets ...................................

Burrell Washington Helton, The solution of a Stieltjes-Volterra integral

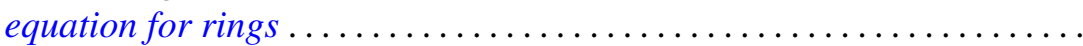

Frank Kwang-Ming Hwang and Shen Lin, Construction of 2-balanced

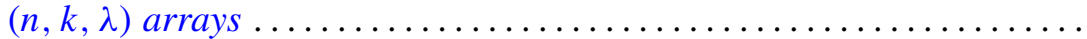

Wei-Eihn Kuan, Some results on normality of a graded ring ... 455

Dieter Landers and Lothar Rogge, Relations between convergence of series and convergence of sequences ......................... 465

Lawrence Louis Larmore and Robert David Rigdon, Enumerating immersions and embeddings of projective spaces ................

Douglas C. McMahon, On the role of an abelian phase group in relativized problems in topological dynamics..................

Robert Wilmer Miller, Finitely generated projective modules and TTF classes...

Yashaswini Deval Mittal, A class of isotropic covariance functions ...

Anthony G. Mucci, Another martingale convergence theorem ...

Joan Kathryn Plastiras, Quasitriangular operator algebras ...

John Robert Quine, Jr., The geometry of $p\left(S^{1}\right) \ldots \ldots \ldots$. 